\title{
Conservation and functional influence of alternative splicing in wood formation of Populus and Eucalyptus
}

Peng $\mathrm{Xu}^{\dagger}$, Yimeng Kong ${ }^{\dagger}$, Dongliang Song, Cheng Huang, Xuan Li and Laigeng Li

\begin{abstract}
Background: Wood formation in tree species is regulated by multiple factors at various layers. Alternative splicing (AS) occurs within a large number of genes in wood formation. However, the functional implications and conservation of the AS occurrence are not well understood.

Results: In this study, we profiled AS events in wood-forming tissues of Populus and Eucalyptus, and analyzed their functional implications as well as inter-species conservation. $28.3 \%$ and $20.7 \%$ of highly expressed transcripts in the developing xylem of Populus and Eucalyptus respectively were affected by AS events. Around $42 \%$ of the AS events resulted in changes to the original reading frame. 25.0\% (in Populus) and 26.8\% (in Eucalyptus) of the AS events may cause protein domain modification. In the process of wood formation, about $28 \%$ of AS-occurring genes were putative orthologs and 71 conserved AS events were identified in the two species.

Conclusion: Through analysis of AS events in developing xylem of two tree species, this study reveals an array of new information regarding AS occurrence and function in tree development.
\end{abstract}

Keywords: Alternative splicing (AS), Comparative transcriptome, Wood formation, Conservation, Populus, Eucalyptus

\section{Background}

Alternative splicing (AS), which generates multiple transcript variants from one gene, is a key scheme in multicellular eukaryotes to enhance the functional diversity of the proteome [1]. In flowering plant species, up to $61 \%$ of multiexonic genes in Arabidopsis and 48\% of genes in rice are affected by AS $[2,3]$. AS has been shown to regulate plant development in photosynthesis, disease resistance, floral transition, circadian rhythm, starch metabolism, auxin biosynthesis, and temperature response [4-6]. AS can cause alterations to protein function through modulating protein structure. For example, frame shifts caused by AS often result in truncated proteins that can form nonfunctional heterodimers and act as dominant-negative regulators $[7,8]$. AS occurrence in functional domain structures can affect protein-protein interaction, transcriptional activation or DNA binding [9-11]. In addition to modulate protein

\footnotetext{
* Correspondence: Igli@sibs.ac.cn

†Equal contributors

National Key Laboratory of Plant Molecular Genetics and Key Laboratory of Synthetic Biology, Institute of Plant Physiology and Ecology, Chinese Academy of Sciences, 300 Fenglin Rd, Shanghai 200032, China
}

functions, AS can influence transcript stability through nonsense-mediated decay (NMD) or miRNA linked regulation $[12,13]$.

Wood, a unique structural and storage tissue in tree species, is derived from the meristematic activities of the vascular cambium during secondary growth $[14,15]$. Wood formation involves a coordinated progression of cell differentiation, expansion, secondary cell wall formation, and programmed cell death [16]. AS has been found to play important roles in the process of wood formation. NAC transcription factor PtrWND1B / PtrSND1-A2, which controls secondary wall biosynthesis, was shown to undergo AS in Populus $[17,18]$. The splice variant of PtrWND1B/ PtrSND1-A2 ${ }^{I R}$ contains a retained intron and encodes a protein lacking DNA binding and transactivation activity but retaining dimerization ability. PtrSND1-A2 $2^{\mathrm{IR}}$ functions as a dominant negative of PtrSND1 members through heterodimerization [17]. This dominant negative regulation was demonstrated as a specific mechanism controlling fiber cell wall thickening during wood formation in Populus [18]. AS may have evolved as a regulatory mechanism of the 
isochorismate synthase gene, PtiICS, which encodes an enzyme for phylloquinone biosynthesis, in Populus [19].

Analyzing how AS is conserved across species can help to identify AS events important in general for plant development. Conserved AS events in several gene families such as ribulosebisphosphate carboxylase/oxygenase activase, SR protein, MYB transcription factor and transthyretin-like protein have been characterized in plant species [20-23]. Genome-wide analysis have identified 56 conserved AS events between Arabidopsis and rice, and 49 conserved AS events between rice and maize [24]. Between two or more legume species, 22 conserved AS events were detected [25]. More conserved AS events (527 cases) were reported between closely related species Brassica and Arabidopsis [26]. Meanwhile, it is not known how AS events are conserved and involved in modulating protein function in wood formation of tree species.

Recently, transcriptome of Populus xylem was analyzed to identify AS events in wood formation tissue. Up to $36 \%$ of expressed genes were detected to undergo AS [27], indicating a wide influence of AS in the process of wood formation. On the other hand, great variations of AS events, which are likely caused by genotypic polymorphism, were observed among different Populus populations [27]. Although AS has shown to influence diverse groups of genes in Populus, how the AS events are involved in protein function modulation is little known. In this study, we compared the occurrence of AS in Eucalyptus and Populus and analyzed the functional implications and conservation of AS during wood formation in tree species.

\section{Results}

\section{AS profiles in wood formation tissues}

To detect AS events during wood formation, we first constructed transcriptomes of developing xylem in Populus (P. × euramericana) and Eucalyptus (E. grandis). Two biological replicates were sampled from the two species for transcriptome analysis using the Illumina platform based RNA-seq technology [28]. The sequencing analysis generated approximate $30 \sim 45$ million paired-end reads (100-nucleotide length) from each sample of the two species. $77.6 \% \sim 80.6 \%$ of these reads could be mapped onto the reference genome via the TopHat program [29]. More than $60 \%$ of the mapped reads located in the exon regions of the two genomes (Additional file 1). In Populus, $26.2 \%$ of the reads were mapped to an exon-intron/intronexon junction region, among which $21.3 \%$ were not annotated in the reference genome. This part of reads could be from the products caused by AS variants. In Eucalyptus, $36.8 \%$ of the junction reads had no annotations. Some reads were found to map to intergenic regions. $6.8 \%$ of the total reads in Eucalyptus were mapped to the intergenic regions of the genome while $0.9 \%$ of reads in Populus showed such mapping. This may reflect the genome annotation difference in two species.

Expressed transcripts were assembled by the Cufflinks program with the cutoff of 0.1 FPKM in both biological replicates [30]. Considering that the weakly expressed transcripts could result in false AS identification [2], highly expressed transcripts with an expression level higher than $5 \%$ of the most abundant transcript were subsequently used as the basis for follow up analysis. In Populus, 31,984 highly expressed transcripts were assembled. Among them, 13.8\% $(4,397)$ of transcripts had no annotation in the reference genome (Additional file 1). In Eucalyptus, the number of highly expressed transcripts was 21,372 and $19.5 \%$ of them were not annotated in the genome. The assembled transcripts were transcribed from 23,735 (57.4\% of the total predicted genes) genes in the Populus genome and 17,202 ( $47.3 \%$ of the total predicted genes) genes in the Eucalyptus genome (Additional files 1 and 2), suggesting that a large portion of the genes in the two species are expressed in developing xylem tissue.

On the basis of the assembled transcripts, AS events among the transcriptome was analyzed. The ASTALAVISTA tool [31] was used to establish the repertoire of AS events. In total, 6,031 AS events in Populus and 2,987 events in Eucalyptus were detected in developing xylem tissue (Additional file 3). These AS events affected $28.3 \%$ and $20.7 \%$ of the highly expressed transcripts in the two species (Figure 1A), respectively, which were transcribed from $17.2 \%$ (4,079 in Populus) and $11.9 \%$ (2,039 in Eucalyptus) of the xylem expressed genes. The majority of AS transcripts (93\% in Populus and 97\% in Eucalyptus) displayed expression levels higher than 1.0 FPKM (Figure 1B). Over $85 \%$ of the AS events occurred in the transcript isoforms with expression ratios of $0.1 \sim$ 1.0 compared to the major transcript of a same gene (Additional file 4). For verification of the AS events, 16 representative AS events were selected for RT-PCR analysis and 14 of them (about 87.5\%) were confirmed (Additional file 5), indicating the reliability of the AS detection in our RNA-seq analysis.

The constructed AS events were categorized into five major AS types [32]: intron retention (IR), alternative acceptor site (AltA), alternative donor site (AltD), exon skipping (ES) and alternative position (AltP) for further analysis. IR, which encompassed $45.0 \%$ and $33.9 \%$ of the AS events in Populus and Eucalyptus respectively (Figure 1C), represented a much higher portion of AS events than ES. AltA was over-represented and IR under-represented while the proportions of the other three AS types were similar in Eucalyptus compared to Populus (Figure 1C).

\section{AS features in wood formation}

Based on the identified AS events in Populus and Eucalyptus, AS features in woody tissues were analyzed, including 


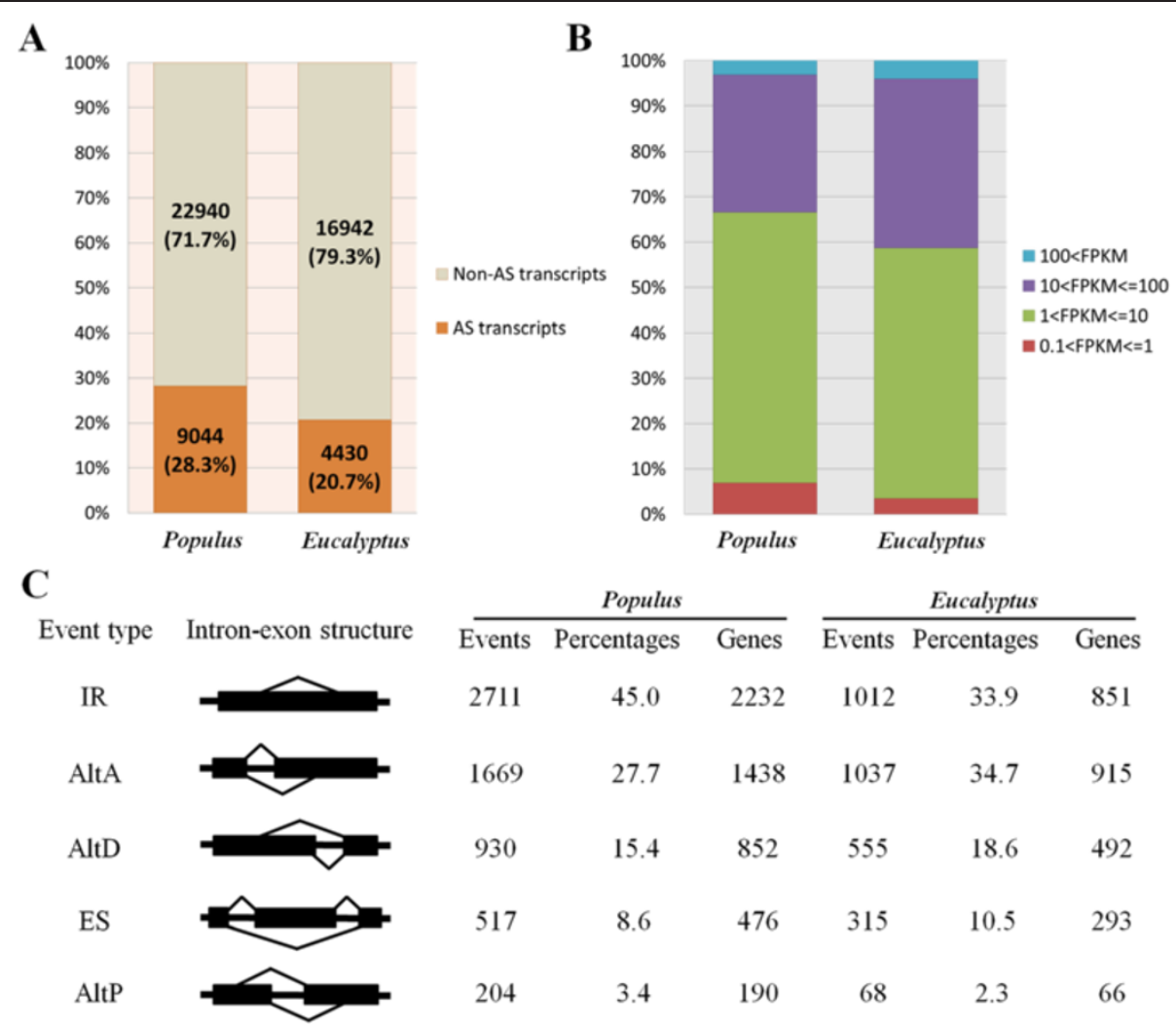

Figure 1 AS profiles in developing xylem of Populus and Eucalyptus. (A) Percentages of expressed transcripts undergoing AS in two species. (B) Expression levels of AS transcript isoforms. The expression abundance was measured with unit of FPKM (Fragments Per Kilobase of transcript per Million mapped reads). (C) Distribution of different AS types. IR, intron retention; AltA, alternative acceptor site; AltD, alternative donor site; ES, exon skipping; AltP, alternative position.

AT content of the introns, and nucleotide change caused by AS. In Populus, the average AT content of the total introns of xylem expressed genes is $66.4 \%$, higher than $62.4 \%$ in Eucalyptus ( $p$-value $<0.0001, t$-test). Similarly, the average AT content across all alternative introns (AS introns) in Populus was 65.6\%, higher than that in Eucalyptus (61.1\%) (Figure 2A). This difference between species was also consistent in the introns among various AS types. Nevertheless, the nucleotide composition at splicing junction site was conserved between Populus and Eucalyptus (Additional file 6).

Selection of different splice sites may result in a string of nucleotides changed between transcript variants. The changed nucleotides could interrupt the original reading frame if they are not multiples of 3 base pairs. About $67 \%$ of the AS events were found to be located in the open reading frame (ORF) region of the two species. Analysis of ORF located AS events revealed that $79 \%$ of the changed nucleotides were between 1 and 200 bps (Figure 2B). A change of 3 nucleotides occurred most frequently in both Populus and Eucalyptus. Similar phenomenon was reported in Arabidopsis, rice and Brassica [26,33]. The 3 nucleotide change was mainly generated from AltA, AltD and AltP, while IR or ES largely caused changes of 50150 bp (Additional file 7). We observed 37\% of the changed lengths fitting in multiples of 3 nucleotides in the two species, which could maintain the original reading frame. In other words, up to $63 \%$ of ORF located AS events (42\% of the total AS events) in the two species could cause frameshift. A similar frequency of ORF change was observed in the AS isoforms with different expression ratios (Additional file 4).

\section{Protein domain modification caused by AS}

One of the major effects of AS is to create protein isoforms that increase proteome diversity [5,34]. Previous studies suggest that protein functions could be affected if their functional domains are interrupted by AS $[9-11,17,18]$. We conducted in silico transcript translation and then analyzed whether AS occurrence caused protein domain alteration. In the two species, $25.0 \%$ (1507 events in Populus) and 26.8\% (801 events in Eucalyptus) of the AS events occurred within domain sequences which would cause domain modification (Additional file 8). A similar frequency of such domain modification was observed in the AS isoforms with different expression ratios (Additional file 4). In detected 

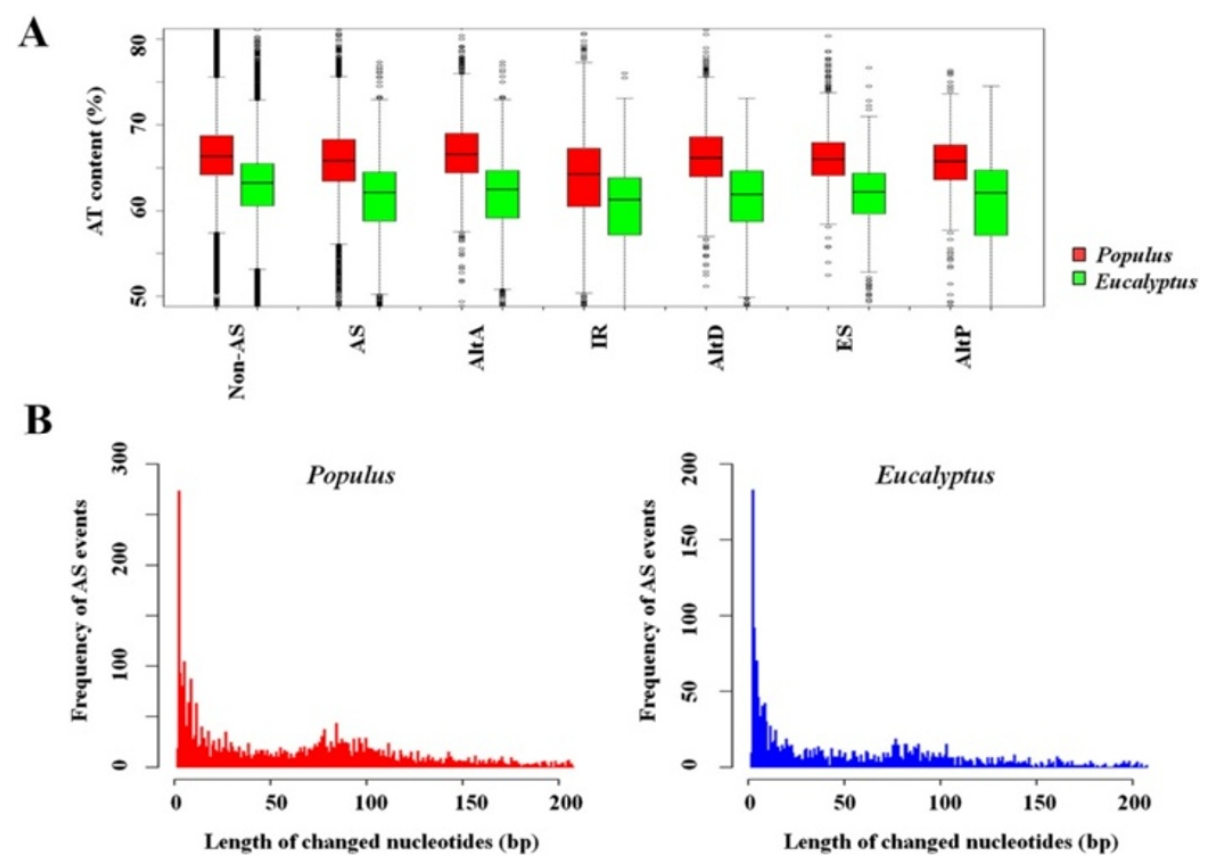

Figure 2 AS profile features in developing xylem of Populus and Eucalyptus. (A) Comparison of AT content in constitutive introns (non-AS introns) and different types of alternative introns (AS introns). IR, intron retention; AltA, alternative acceptor site; AltD, alternative donor site; ES, exon skipping; AltP, alternative position. (B) Histogram indicating frequency of ORF located AS events at different changed lengths of nucleotides.

AS events, 789 and 500 kinds of domains might be modified in Populus and Eucalyptus, respectively. Moreover, 16.6\% (1003 events in Populus) and 18.2\% (545 events in Eucalyptus) of AS events could result in domain gain or loss. Among the 15 most frequently modified domains, 8 domains were present in both Populus and Eucalyptus, namely Pkinase, Pkinase_Tyr, RRM_1, PP2C, zf-DHHC, Ras, Ndr and DnaJ (Figure 3A). To exclude the potential influence of domain number in the background genome, Fisher's exact test was used to obtain the enriched domains in the AS affected genes (Additional file 9). 51 enriched domains showed a close association with AS modification in the two species $(p$-value $<0.05)$, including domains RRM_1, PP2C and Ndr which were identified among the most frequently modified domains. This suggests that these protein domains may be particularly modified by AS events in wood formation process.

To understand how AS impacts wood formation, the AS modified domains from Populus and Eucalyptus were mapped to individual genes associated with wood formation according to their annotation (Additional file 10). These genes could be categorized into five fundamental processes for wood formation including: cell wall formation, hormone signaling, transcriptional regulation, cellular transportation and programmed cell death (Figure 3B). For example, cellulose synthase (CESA) and caffeoyl-CoA 3-Omethyltransferase (CCOAMT) are involved in secondary cell wall thickening during wood formation [35-37]. The occurrence of AS was observed in the catalytic domains of these enzymes. The functional domains of many other wood formation-related genes was also detected to be affected by AS.

\section{Conservation of AS-occurring genes in wood formation}

To investigate conservation of AS-occurring genes, putative orthologous genes in Populus and Eucalyptus were searched by the BLAST program (E-value $<1 \mathrm{e}-50$, identity $>0.4$ and coverage $>0.6$ ). A total of 1159 Populus and 887 Eucalyptus putative orthologous genes were identified with AS-occurrence, which represents $28 \%$ and $44 \%$ of the AS-occurring genes in the two species, respectively (Figure 4A). Gene expression patterns established via microarray analysis [38,39] revealed that $56.3 \%$ of these orthologous genes were highly expressed in xylem (Figure 4B), indicating their association with wood formation. These orthologs in the two species constituted 716 independent gene groups with different biological functions (Additional file 11). Gene ontology (GO) annotations suggested several important processes for plant development such as transportation, cell cycle and cell growth were significantly enriched (FDR < 0.001) (Figure 4C). Genes involved in the process responding to abiotic stimulus were also enriched, highlighting the potential impact of environmental cues on AS-occurring genes.

\section{Conserved AS events in wood formation}

Among orthologous genes with AS occurrence, conserved introns were searched to identify conserved AS 


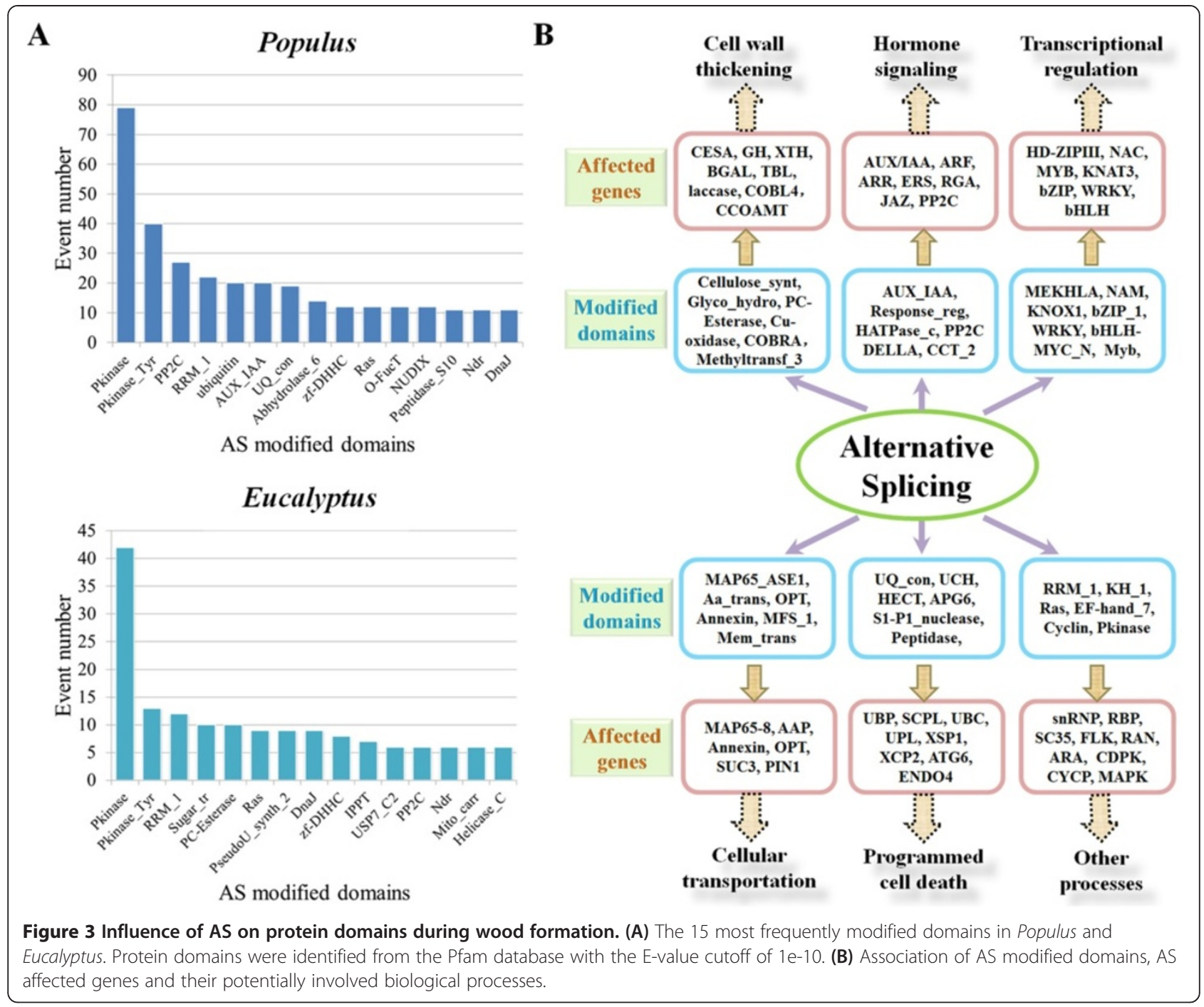

events across the two species. A total of 71 conserved AS events (1.2\% of total AS events in Populus and $2.4 \%$ in $\mathrm{Eu}$ calyptus) were identified. These conserved AS events occurred between 65 Populus and 55 Eucalyptus genes (Additional file 12). Among them, 37 events belonged to the AltA type, ranking it as the most frequent type of AS. Approximately, $60 \%$ of the conserved AS events caused frameshift and $48 \%$ of the conserved AS events were detected to cause modification of functional domain structures (Additional file 12).

Two examples of the conserved AS events in the two species were further analyzed in greater detail. AS was observed in an orthologous pair of AP2 genes in Populus and Eucalyptus (Figure 5A). The AP2/EREBP family encodes transcription factors that play a role in a variety of plant regulatory processes [40]. The AP2 domain of this gene family consists of 60 amino acids that are responsible for DNA binding. In Populus, one isoform of the PtAP2 transcript has two AP2 domains while the second isoform only has one AP2 domain due to a skipped exon. The conserved AS event was also observed in Eucalyptus. Another example of conserved AS event occurred in the functional domain of Aux/IAA gene family, which encode short-lived nuclear proteins that negatively regulate auxin signaling transduction [41]. Aux/IAA proteins are likely involved in the early response to auxin signaling, which controls the development of wood-forming tissues [42]. Based on our analysis, a conserved intron is retained in the IAA8 genes of Populus and Eucalyptus. Importantly, the conserved IR event resulted in a truncated protein as it introduced a premature termination codon in the transcript (Figure 5B). RT-PCR verified the two cases of conserved AS events in the two species (Figure 5C).

To study how AS can regulate the function of the Aux/ IAA family, sequences of IAA8 orthologs from Arabidopsis, rice, Populus and Eucalyptus were aligned. Multiple sequence alignment showed the truncated proteins, PtIAA $8^{I R}$ and EgIAA $88^{I R}$, specifically lack the conserved 


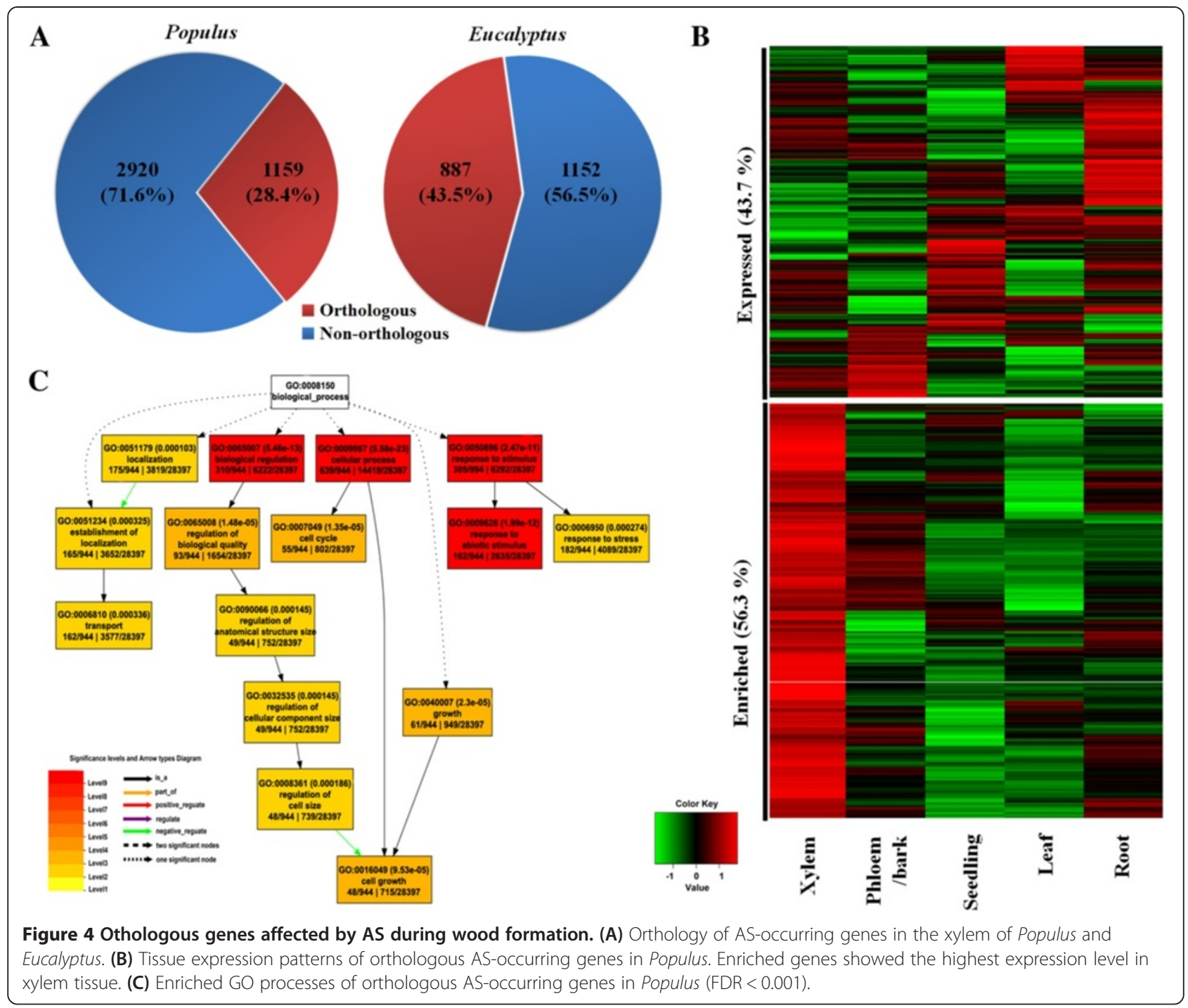

subdomain IV of Aux/IAA proteins, but contain intact subdomains I $\sim$ III (Figure 6 A). Subdomain IV contains a sequence which is critical for nucleus localization [43]. To examine whether the location of the protein is affected by AS, IAA8 isoforms from Populus were fused with GFP for localization analysis. When expressed in tobacco leaves, the protein encoded by full transcript PtIAA8 was shown to locate in the nucleus, while the truncated protein PtIAA $8{ }^{\mathrm{IR}}$ displayed ubiquitous location in cells (Figure 6B). This indicates AS can modulate protein functions of Aux/IAAs through regulating their intercellular location.

\section{Discussion}

\section{Landscape of AS in wood formation of tree species}

This study established a comprehensive profile of AS events during wood formation. The results show that in both Populus and Eucalyptus more than 20\% of the highly expressed transcripts were affected by AS, indicating extensive influence of AS on wood formation. In Populus, 6,031 AS events were detected while 2,987 events were detected in Eucalyptus. Based on current information, however, it is unknown whether this difference is implicated by the characteristics of the species. Five AS types were detected but their composition ratio was different in the two species. AltA was more frequently in Eucalyptus while IR was observed with a relatively greater frequency in Populus. Interestingly, intron sequences in Eucalyptus have a significantly lower AT content compared to those in Populus. Variations of AT content may be responsible for the different AS profiles in the two species, as UArichness of plant introns is important for splicing efficiency [44]. AS identification in the developing xylem of Populus trichocarpa has recently reported [27]. About 7 $11 \%$ of the total annotated genes were found to undergo AS in 20 Populus individuals from different populations, while around $46 \%$ of the AS events were not conserved 
A

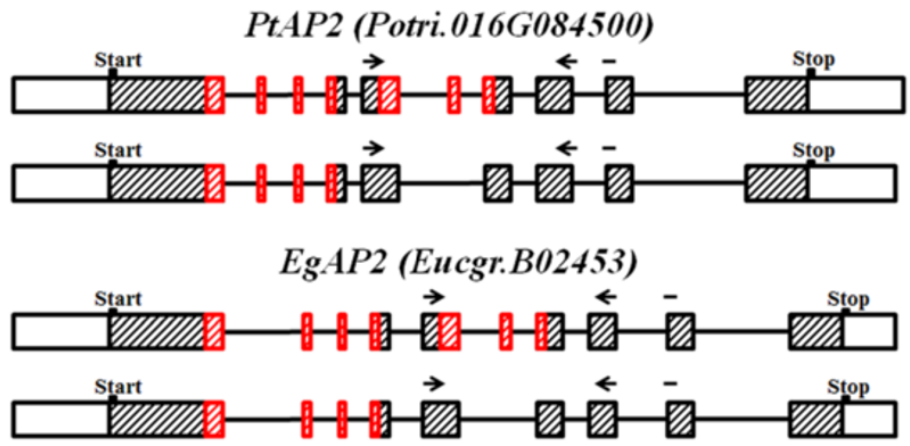

AP2

B

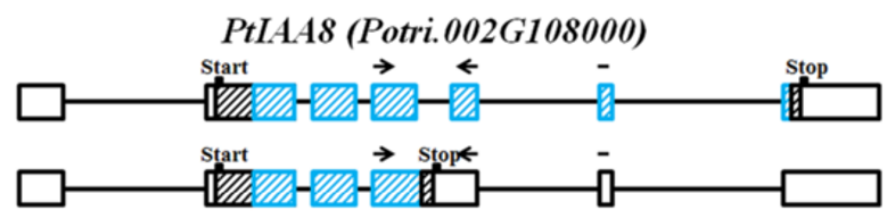

EgIAA8 (Eucgr.F02172)

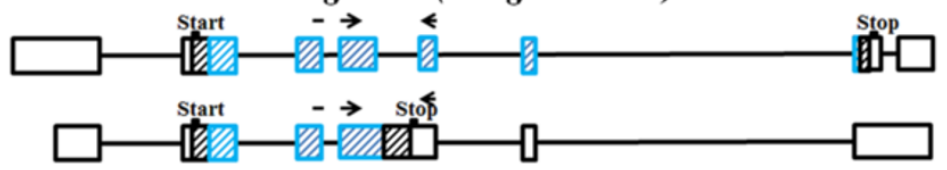

QAUX/LAA

C

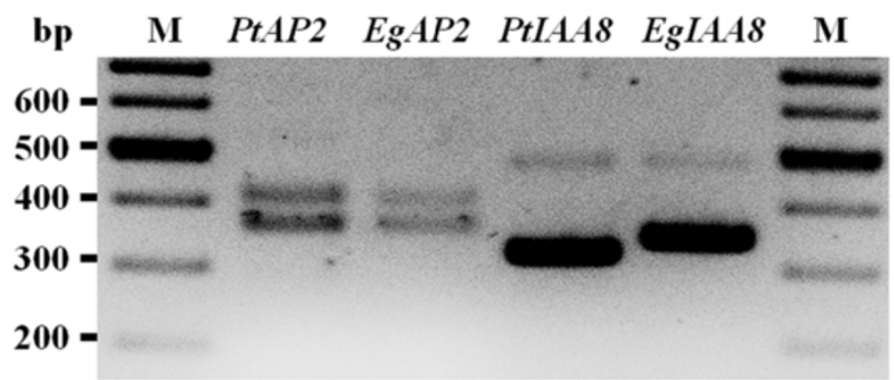

Figure 5 Characterization of two conserved AS events in developing xylem of Populus and Eucalyptus. Gene structures are shown for the conserved AS events in AP2 family (A) and AUX/IAA family (B). Boxes represent exons and lines represent introns. Hatched regions indicate coding sequences. Red and blue colors indicate predicted domains. Primers designed for RT-PCR are shown by black arrows. (C) RT-PCR verification of the two conserved AS events. The upper bands indicate the full transcripts in AP2 lanes and the intron-retained transcripts in AUXIAA lanes.

within individuals, which may be caused by genotypic variations [27]. In the present study, the occurrence of AS was detected in about $10 \%$ of the total annotated genes and about $40 \%$ of the AS-occurring genes were the same in the reported individuals (PT17 and PT18) [27], indicating a consistency of AS occurrence in different Populus species. The results may also reflect some of the detected AS events being specific to the gene type of hybrid poplars (P. $\times$ euramericana $c v$. Nanlin895').
Functional implications of AS in wood formation

AS occurs extensively in various plant species, however, comprehensive analysis of their functional impact has been little studied. AS affect protein functions in a variety of ways by altering amino acid composition, subcellular location, secondary structure stability, binding property, and posttranslational modification [45]. The diversified aspects of AS influence can be assessed through in silico analysis. In this study, we primarily focused on analysis of AS- 


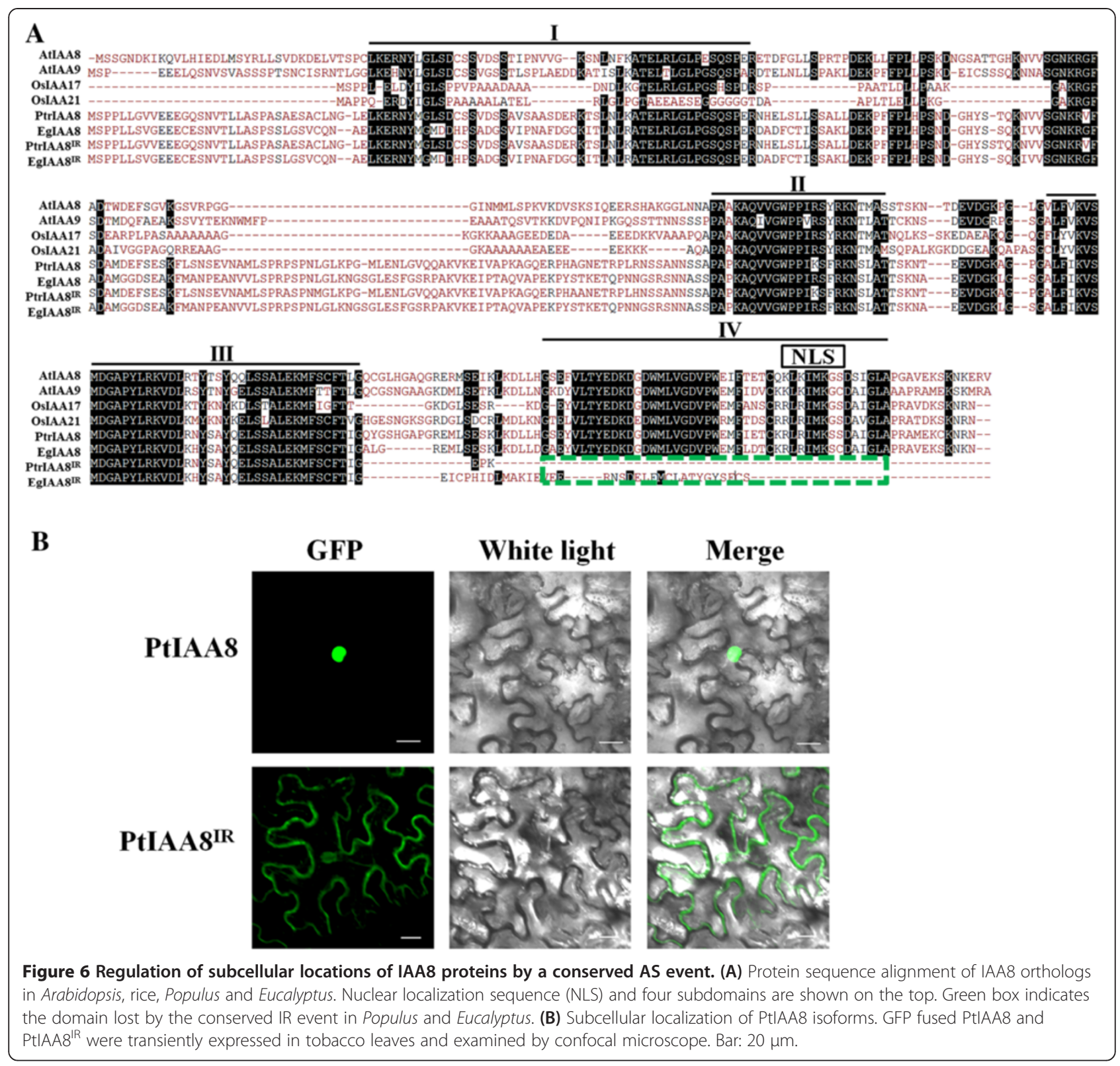

occurring genes which bring about modification of domain structures. Results show that about $42 \%$ of the AS events caused ORF disruptions or change, suggesting that AS extensively affects protein sequence. A large proportion (25.0\% in Populus and 26.8\% in Eucalyptus) of AS events resulted in protein domain modifications, which may be involved in wood formation at various levels. For example, the protein domains modified included Cellulose_synt, Glyco_transf and Glyco_hydro, which function in cell wall biosynthesis. Protein domains like proteasome and peptidase are involved in programmed cell death. AS modification to the AUX/IAA and PP2C domains indicates that AS may play a role in auxin and ABA signaling in wood formation tissue. We also observed a similar pattern of ORF change or domain modification in the AS events with different isoform ratios.

Apart from generating functionally tailored proteins, AS could also affect mRNA transport, localization and stability prior to protein translation. For example, some intron-containing transcript variants could remain within the nucleus resulting in decreased mobility [46]. This indicates a subset of AS transcripts which cannot be translated into proteins as in silico predictions would suggest. In addition, aberrant transcripts caused by AS are often degraded by RNA surveillance pathways such as nonsensemediated decay (NMD) [12]. In theory, NMD sensitive transcripts would generally display low expression levels. A group of such AS events might be excluded from this study 
as weakly expressed transcripts were filtered out in analysis. Thus, a normalized cDNA library should be constructed in order to estimate NMD targets during wood formation [2]. Intriguingly, typical NMD targets in Arabidopsis include mRNA with a 3'UTR sequence longer than 350 bp [47-49]. Our analysis suggests that the average length of 3'UTR in both Populus and Eucalyptus is over 400 bp. And 3'UTR sequences are usually longer than $700 \mathrm{bp}$ when they contained AS events. This may suggest a difference between Arabidopsis and tree species in the precise length of 3'UTR that could trigger NMD pathway. Taken together, our results indicate that the AS events may function in regulating wood formation through modifying protein domain structures.

\section{Conservation of AS in Populus and Eucalyptus}

By comparing AS profiles in Populus and Eucalyptus, this study provides a sketch of AS events conserved in wood formation tissue. In our results, AS occurred within 1159 Populus and 887 Eucalyptus putative orthologous genes. GO analysis revealed that the orthologous genes were featured with functions related to the regulation of cell growth and differentiation. Over half of the orthologous genes were preferentially expressed in the developing xylem of Populus, indicating their involvement in wood formation.

Comparison of AS events identified 71 AS events that were conserved in Populus and Eucalyptus. Among them, $47.9 \%$ of the conserved AS events caused domain modifications. For instance, a conserved IR event was demonstrated to affect subdomain IV of Aux/IAA isoforms, which is critical to regulate protein subcellular location. The intron-retained $A u x / I A A$ isoforms contain a premature termination codon and have a long 3'UTR sequence (>700 bp), suggesting they may be regulated by the NMD pathway that is coupled with AS occurrence. Consistent with this, these transcripts displayed reduced expression level according to RT-PCR. Interestingly, no equivalent AS regulation has been reported for $A u x / I A A$ family genes in other plant species such as Arabidopsis and rice. Whether this AS event regulation of $A u x / I A A$ family genes is specific to wood formation is yet to be further characterized.

\section{Conclusions}

AS plays a critical role in the development of multicellular organisms, though much remains to be learned about its function in tree-specific processes. AS is extensively involved in wood formation as revealed by profiles of AS-occurring genes, AS events and AS modification of protein structures in the developing xylem of Populus and Eucalyptus. The results of this study highlight a number of new avenues to explore how AS is employed as a mechanism to modulate gene functions in woodforming tissues.

\section{Methods}

\section{RNA-seq experiment}

The Populus (P. $\times$ euramericana cv.'Nanlin895') and Eucalyptus ( $E$. grandis) were grown in experimental fields in Shanghai $\left(31^{\circ} \mathrm{N}\right)$ and Guangxi Province $\left(21^{\circ} \mathrm{N}\right)$. Developing xylem from 3-year old trees was sampled at midday (June, 2012) and razor blades were used to collect xylem tissues after peeling off the bark. Xylem tissues from three individual trees of each species were mixed together for sample preparation. Two biological replicates of xylem samples in each species were used for RNA extraction following the CTAB method. RNA concentration and quality were determined by the Agilent Bioanalyzer 2100. The cDNA library was constructed by polyA enriched RNA. 200-300 bp fragments were selected for non-directional 100 nucleotide paired-end sequencing, which was performed on an Illumina HiSeq 2000 according to Illumina protocols (San Diego, CA, USA).

\section{Detection of AS events}

The reads from two biological replicates in each species were mapped and assembled independently. Reference genomes of Populus (v3.0) [50] and Eucalyptus (v1.1) [51] were obtained from Phytozome database. RNA-seq reads were mapped onto reference genomes via TopHat (v2.0.10) [29]. The maximum intron length was set to $6000 \mathrm{bp}$ and other parameters were left as defaults. Unique mapped reads were extracted by samtools (v0.1.18) with a MAPQ cutoff of 20 [52]. Cufflinks (v2.1.1) was used for transcript assembly with supplied genome annotations [30]. Expression abundance of assembled transcripts was obtained from the Cufflinks output, which was measured in the unit of FPKM (Fragments Per Kilobase of transcript per Million mapped reads). Expression of $99 \%$ of the transcripts were consistent between two replicates in each species (FDR $<0.05)$, indicating a good reproducibility. Downstream analysis are based on the genes that could be detected by RNA-seq (>0.1 FPKM) in both biological replicates. To ensure the reliability of AS detection, weakly expressed transcripts with expression levels less than $5 \%$ of the most abundant transcript format from a gene, were filtered out before AS detection. Transcripts that remained in both replicates were subjected to ASTALAVISTA web server to discover AS events [31]. AS transcripts were extracted from each AS event and the percentage of AS transcripts was calculated through dividing the number of AS transcripts by that of the total transcripts. Within a gene, isoform ratio of the AS event was set as the expression level of the minor transcript divided by that of the most abundant transcript. Five major types of AS previously described in Wang et al. 2006 [32], including intron retention (IR), alternative acceptor site (AltA), alternative donor site (AltD), exon skipping (ES) and alternative position (AltP), were extracted for further analysis. 


\section{Analysis of AS features}

Intron sequences with different AS types were extracted according to the coordinates reported by ASTALAVISTA. Transcript isoforms were translated based on the longest ORF. AS events were classified as 5'UTR, ORF and 3'UTR location according to their positions in different mRNA features. Perl scripts were written for analysis including AT content and changed nucleotides. The length of changed nucleotides was computed by comparing the alternative introns from two isoforms in a single AS event. For IR and ES, it was the length of the retained intron and skipped exon. For AltA, AltD and AltP, the length corresponded to the altered nucleotides that were present in the exon of one isoform, but included in the intron of the other isoform. The Pfam 27.0 database was searched to identify the domains affected by AS, with an E-value cutoff of 1e-10. The genes with AS modified domains were examined for their function annotations (on the basis of information in Phytozome) and classified into different biological processes. To determine significantly enriched protein domains associated with the AS occurrence, Fisher's exact test was conducted with a $p$-value cutoff of less than 0.05 .

\section{Conservation analysis of AS-occurring genes}

Orthology of the AS-occurring genes in Populus and Eucalyptus was investigated by the BLAST program (v2.2.21) with parameters of E-value $<1 \mathrm{e}-50$, identity $>$ 0.4 and coverage $>0.6$. Expression patterns of the orthologous genes in Populus were established by microarray data from the NCBI Gene Expression Omnibus (GEO) database under accession numbers GSE30507 and GSE13990. Robust Multiarray Averaging (RMA) method was used to normalize microarray data across different tissues in Bioconductor according to previously described methods [53]. Enriched genes were defined as those with the highest expression level in xylem, compared to other tissues. The other genes were included as expressed genes. Heatmap. 2 in R (v2.15.1) program was used to generate heatmaps of enriched expressed genes. Populus genes were converted to their orthologous genes in Arabidopsis and then used for GO annotation by agriGO [54]. Plant GOslim database and Fisher's exact test were implemented to identify enriched GO processes $($ FDR $<0.001)$.

\section{Identification of conserved AS events}

Similar to previous reports [24,32], conserved AS events in the two species were defined mainly by identification of the conserved introns in orthologous AS genes. To get conserved introns, the introns flanked by homologous exonic sequences were searched. In every AS event from Populus and Eucalyptus, 100 bp exonic sequences flanking the alternative introns were extracted. The tblastx program was used to search homologous exonic sequences across the two species with an E-value cutoff of 1e-5. If both of the flanking exonic sequences of a Populus intron were homologous to that of a Eucalyptus intron, the alternative introns were regarded as conserved introns. If the conserved introns were present in orthologous genes with the same AS type, the pair of AS event was considered a conserved AS event.

\section{Subcellular localization and RT-PCR analysis}

Coding sequences of PtIAA 8 and PtIAA ${ }^{I R}$ were cloned into pCAMBIA2300 backbone fused in frame with GFP under the control of the CaMV 35S promoter to determine their subcellular location. Tobacco leaves were transformed with constructs mediated with Agrobacterium strain GV3101 according to previous descriptions [55]. GFP fused proteins were examined with a confocal microscope (LSM 510 META; Zeiss) using $488 \mathrm{~nm}$ for excitation and 505 to $555 \mathrm{~nm}$ for emitted light capture. For RT-PCR, the xylem of Populus (P. $\times$ euramericana) and Eucalyptus (E. grandis) was used for RNA extraction. $500 \mathrm{ng}$ RNA was reverse transcribed into cDNA by PrimeScript RT Master Mix (TaKaRa, Dalian, China) according to the manufacturer's protocols. Primers that span alternative introns were designed to perform PCR reactions in each species (Additional file 13).

\section{Availability of supporting data}

All sequencing data were deposited in the Short Read Archive at NCBI under accession number SRA108028.

\section{Additional files}

Additional file 1: Summary of reads mapping and transcript assembly.

Additional file 2: Assembled transcripts in Populus and Eucalyptus. Additional file 3: AS events in Populus and Eucalyptus.

Additional file 4: Functional influences of AS events in different isoform ratios.

Additional file 5: Confirmation of AS events by RT-PCR.

Additional file 6: Nucleotide conservation at the junction sites of xylem expressed genes.

Additional file 7: Changed nucleotides in different types of alternative splicing.

Additional file 8: AS modified domains in Populus and Eucalyptus. Additional file 9: Domain enrichment in AS genes.

Additional file 10: AS modified domains in wood formation-related genes.

Additional file 11: Orthologous groups of AS-occurring genes.

Additional file 12: Conserved alternative splicing events in Populus and Eucalyptus.

Additional file 13: Primers designed for RT-PCR confirmation.

Competing interests

The authors declare that they have no competing interests. 


\section{Authors' contributions}

PX designed the study, conducted the experiments and prepared the manuscript. YK performed the statistical analysis and participated in the design of the study. LL directed the research project and composed the manuscript. XL directed data analysis and edited the manuscript. DS prepared the tissue samples. $\mathrm{CH}$ participated in the confocal microscopy analysis. All the authors have read and approved the final manuscript.

\section{Acknowledgments}

This work was supported by the National Key Basic Research Program of China (2012CB114502), the National Natural Science Foundation of China (31130012), and the National Natural Science Foundation of China (31271409).

Received: 26 April 2014 Accepted: 8 September 2014

Published: 10 September 2014

\section{References}

1. Graveley BR: Alternative splicing: increasing diversity in the proteomic world. Trends Genet 2001, 17(2):100-107.

2. Marquez Y, Brown JWS, Simpson C, Barta A, Kalyna M: Transcriptome survey reveals increased complexity of the alternative splicing landscape in Arabidopsis. Genome Res 2012, 22(6):1184-1195.

3. Lu T, Lu G, Fan D, Zhu C, Li W, Zhao Q, Feng Q, Zhao Y, Guo Y, Huang X, Han B: Function annotation of the rice transcriptome at singlenucleotide resolution by RNA-seq. Genome Res 2010, 20(9):1238-1249.

4. Syed NH, Kalyna M, Marquez $Y$, Barta A, Brown JWS: Alternative splicing in plants - coming of age. Trends Plant Sci 2012, 17(10):616-623.

5. Reddy ASN, Marquez Y, Kalyna M, Barta A: Complexity of the Alternative Splicing Landscape in Plants. Plant Cell 2013, 25(10):3657-3683.

6. Staiger D, Brown JWS: Alternative Splicing at the Intersection of Biological Timing, Development, and Stress Responses. Plant Cell 2013, 25(10):3640-3656.

7. Staudt A-C, Wenkel S: Regulation of protein function by 'microProteins'. EMBO Rep 2010, 12(1):35-42.

8. Seo PJ, Hong S-Y, Kim S-G, Park C-M: Competitive inhibition of transcription factors by small interfering peptides. Trends Plant Sci 2011, 16(10):541-549.

9. Penfield S, Josse E-M, Halliday KJ: A role for an alternative splice variant of PIF6 in the control of Arabidopsis primary seed dormancy. Plant Mol Biol 2009, 73(1-2):89-95.

10. Seo PJ, Kim MJ, Ryu J-Y, Jeong E-Y, Park C-M: Two splice variants of the IDD14 transcription factor competitively form nonfunctional heterodimers which may regulate starch metabolism. Nat Commun 2011, 2:303.

11. Liu J, Sun N, Liu M, Du B, Wang X, Qi X: An Autoregulatory Loop Controlling Arabidopsis HsfA2 Expression: Role of Heat Shock-Induced Alternative Splicing. Plant Physiol 2013, 162(1):512-521.

12. Lewis BP, Green RE, Brenner SE: Evidence for the widespread coupling of alternative splicing and nonsense-mediated mRNA decay in humans. Proc Natl Acad Sci U S A 2003, 100(1):189-192.

13. Yang $X$, Zhang $H$, Li L: Alternative mRNA processing increases the complexity of microRNA-based gene regulation in Arabidopsis. Plant $J$ 2012, 70(3):421-431

14. Plomion C, Leprovost G, Stokes A: Wood formation in trees. Plant Physiol 2001, 127(4):1513-1523.

15. Groover A, Nieminen $K$, Helariutta $Y$, Mansfield S: Wood Formation in Populus. In Genetics and Genomics of Populus. 8th edition. Edited by Jansson S, Bhalerao R, Groover A. New York: Springer; 2010:201-224.

16. Hertzberg M, Aspeborg H, Schrader J, Andersson A, Erlandsson R, Blomqvist K, Bhalerao R, Uhlen M, Teeri TT, Lundeberg J, Sundberg B, Nilsson P, Sandberg G: A transcriptional roadmap to wood formation. Proc Natl Acad Sci U S A 2001, 98(25):14732-14737.

17. Li Q, Lin YC, Sun YH, Song J, Chen H, Zhang XH, Sederoff RR, Chiang VL: Splice variant of the SND1 transcription factor is a dominant negative of SND1 members and their regulation in Populus trichocarpa. Proc Natl Acad Sci U S A 2012, 109(36):14699-14704

18. Zhao Y, Sun J, Xu P, Zhang R, Li L: Intron-Mediated Alternative Splicing of WOOD-ASSOCIATED NAC TRANSCRIPTION FACTOR1B Regulates Cell Wall Thickening during Fiber Development in Populus Species. Plant Physiol 2014, 164(2):765-776.

19. Yuan Y, Chung JD, Fu X, Johnson VE, Ranjan P, Booth SL, Harding SA, Tsai $\mathrm{CJ}$ : Alternative splicing and gene duplication differentially shaped the regulation of isochorismate synthase in Populus and Arabidopsis. Proc Natl Acad Sci 2009, 106(51):22020-22025.
20. Werneke JM, Chatfield JM, Ogren WL: Alternative mRNA splicing generates the two ribulosebisphosphate carboxylase/oxygenase activase polypeptides in spinach and Arabidopsis. Plant Cell 1989, 1(8):815-825

21. Kalyna M, Lopato S, Voronin V, Barta A: Evolutionary conservation and regulation of particular alternative splicing events in plant SR proteins. Nucleic Acids Res 2006, 34(16):4395-4405.

22. Li J: A subgroup of MYB transcription factor genes undergoes highly conserved alternative splicing in Arabidopsis and rice. J Exp Bot 2006, 57(6):1263-1273.

23. Lamberto I, Percudani R, Gatti R, Folli C, Petrucco S: Conserved Alternative Splicing of Arabidopsis Transthyretin-Like Determines Protein Localization and S-Allantoin Synthesis in Peroxisomes. Plant Cell 2010, 22(5):1564-1574.

24. Severing El, van Dijk ADJ, Stiekema WJ, van Ham RCHJ: Comparative analysis indicates that alternative splicing in plants has a limited role in functional expansion of the proteome. BMC Genomics 2009, 10(1):154.

25. Wang B-B, O'Toole M, Brendel V, Young ND: Cross-species EST alignments reveal novel and conserved alternative splicing events in legumes. BMC Plant Biol 2008, 8(1):17.

26. Darracq A, Adams KL: Features of evolutionarily conserved alternative splicing events between Brassica and Arabidopsis. New Phytol 2013, 199(1):252-263.

27. Bao H, Li E, Mansfield S, Cronk Q, El-Kassaby Y, Douglas C: The developing xylem transcriptome and genome-wide analysis of alternative splicing in Populus trichocarpa (black cottonwood) populations. BMC Genomics 2013, 14(1):359.

28. Metzker ML: Sequencing technologies - the next generation. Nat Rev Genet 2009, 11(1):31-46.

29. Trapnell C, Pachter L, Salzberg SL: TopHat: discovering splice junctions with RNA-Seq. Bioinformatics 2009, 25(9):1105-1111.

30. Trapnell C, Williams BA, Pertea G, Mortazavi A, Kwan G, van Baren MJ, Salzberg SL, Wold BJ, Pachter L: Transcript assembly and quantification by RNA-Seq reveals unannotated transcripts and isoform switching during cell differentiation. Nat Biotechnol 2010, 28(5):511-515.

31. Foissac S, Sammeth M: ASTALAVISTA: dynamic and flexible analysis of alternative splicing events in custom gene datasets. Nucleic Acids Res 2007, 35(Web Serve):W297-W299.

32. Wang BB: Genomewide comparative analysis of alternative splicing in plants. Proc Natl Acad Sci 2006, 103(18):7175-7180.

33. Campbell M, Haas B, Hamilton J, Mount S, Buell CR: Comprehensive analysis of alternative splicing in rice and comparative analyses with Arabidopsis. BMC Genomics 2006, 7(1):327.

34. Blencowe BJ: Alternative Splicing: New Insights from Global Analyses. Cell 2006, 126(1):37-47.

35. Ye ZH: Association of Caffeoyl Coenzyme A 3-O-Methyltransferase Expression with Lignifying Tissues in Several Dicot Plants. Plant Physiol 1997, 115(4):1341-1350.

36. Guo D, Chen F, Inoue K, Blount JW, Dixon RA: Downregulation of caffeic acid 3-O-methyltransferase and caffeoyl CoA 3-O-methyltransferase in transgenic alfalfa. impacts on lignin structure and implications for the biosynthesis of $\mathrm{G}$ and S lignin. Plant Cell 2001, 13(1):73-88.

37. Mizrachi E, Mansfield SD, Myburg AA: Cellulose factories: advancing bioenergy production from forest trees. New Phytol 2012, 194(1):54-62

38. Wilkins O, Nahal H, Foong J, Provart NJ, Campbell MM: Expansion and Diversification of the Populus R2R3-MYB Family of Transcription Factors. Plant Physiol 2008, 149(2):981-993.

39. Ko J-H, Kim H-T, Hwang I, Han K-H: Tissue-type-specific transcriptome analysis identifies developing xylem-specific promoters in poplar. Plant Biotechnol J 2012, 10(5):587-596

40. Dietz K-J, Vogel MO, Viehhauser A: AP2/EREBP transcription factors are part of gene regulatory networks and integrate metabolic, hormonal and environmental signals in stress acclimation and retrograde signalling. Protoplasma 2010, 245(1-4):3-14

41. Ouellet F, Overvoorde PJ, Theologis A: IAA17/AXR3: biochemical insight into an auxin mutant phenotype. Plant Cell 2001, 13(4):829-841.

42. Nilsson J, Karlberg A, Antti H, Lopez-Vernaza M, Mellerowicz E, PerrotRechenmann C, Sandberg G, Bhalerao RP: Dissecting the Molecular Basis of the Regulation of Wood Formation by Auxin in Hybrid Aspen. Plant Cell 2008, 20(4):843-855.

43. Reed JW: Roles and activities of Aux/IAA proteins in Arabidopsis. Trends Plant Sci 2001, 6(9):420-425. 
44. Lorkovic ZJ, Wieczorek Kirk DA, Lambermon MH, Filipowicz W: Pre-mRNA splicing in higher plants. Trends Plant Sci 2000, 5(4):160-167.

45. Stamm S, Ben-Ari S, Rafalska I, Tang Y, Zhang Z, Toiber D, Thanaraj TA, Soreq H: Function of alternative splicing. Gene 2005, 344:1-20.

46. Gohring J, Jacak J, Barta A: Imaging of Endogenous Messenger RNA Splice Variants in Living Cells Reveals Nuclear Retention of Transcripts Inaccessible to Nonsense-Mediated Decay in Arabidopsis. Plant Cell 2014, 26(2):754-764.

47. Kerenyi Z, Merai Z, Hiripi L, Benkovics A, Gyula P, Lacomme C, Barta E, Nagy F, Silhavy D: Inter-kingdom conservation of mechanism of nonsense-mediated mRNA decay. EMBO J 2008, 27(11):1585-1595.

48. Kalyna M, Simpson CG, Syed NH, Lewandowska D, Marquez Y, Kusenda B, Marshall J, Fuller J, Cardle L, McNicol J, McNicol J, Dinh HQ, Barta A, Brown JW: Alternative splicing and nonsense-mediated decay modulate expression of important regulatory genes in Arabidopsis. Nucleic Acids Res 2011, 40(6):2454-2469.

49. Kertesz S, Kerenyi Z, Merai Z, Bartos I, Palfy T, Barta E, Silhavy D: Both introns and long $3^{\prime}-U T R s$ operate as cis-acting elements to trigger nonsense-mediated decay in plants. Nucleic Acids Res 2006, 34(21):6147-6157.

50. Tuskan GA, Difazio S, Jansson S, Bohlmann J, Grigoriev I, Hellsten U, Putnam N, Ralph S, Rombauts S, Salamov A, Schein J, Sterck L, Aerts A, Bhalerao RR, Bhalerao RP, Blaudez D, Boerjan W, Brun A, Brunner A, Busov V, Campbell M, Carlson J, Chalot M, Chapman J, Chen GL, Cooper D, Coutinho PM, Couturier J, Covert S, Cronk Q, et al: The genome of black cottonwood, Populus trichocarpa (Torr. \& Gray). Science 2006, 313(5793):1596-1604.

51. Myburg AA, Grattapaglia D, Tuskan GA, Hellsten U, Hayes RD, Grimwood J, Jenkins J, Lindquist E, Tice H, Bauer D, Goodstein DM, Dubchak I, Poliakov A, Mizrachi E, Kullan AR, Hussey SG, Pinard D, van der Merwe K, Singh P, van Jaarsveld I, Silva-Junior OB, Togawa RC, Pappas MR, Faria DA, Sansaloni CP, Petroli CD, Yang X, Ranjan P, Tschaplinski TJ, Ye CY, et al: The genome of Eucalyptus grandis. Nature 2014, 510(7505):356-362.

52. Li H, Handsaker B, Wysoker A, Fennell T, Ruan J, Homer N, Marth G, Abecasis G, Durbin R: The Sequence Alignment/Map format and SAMtools. Bioinformatics 2009, 25(16):2078-2079.

53. Zhang Y, Szustakowski J, Schinke M: Bioinformatics analysis of microarray data. Methods Mol Biol 2009, 573:259-284.

54. Du Z, Zhou X, Ling Y, Zhang Z, Su Z: agriGO: a GO analysis toolkit for the agricultural community. Nucleic Acids Res 2010, 38(Web Server issue):W64-W70

55. Vaghchhipawala Z, Rojas CM, Senthil-Kumar M, Mysore KS: Agroinoculation and agroinfiltration: simple tools for complex gene function analyses. Methods Mol Biol 2011, 678:65-76.

doi:10.1186/1471-2164-15-780

Cite this article as: Xu et al:: Conservation and functional influence of alternative splicing in wood formation of Populus and Eucalyptus. BMC Genomics 2014 15:780.

\section{Submit your next manuscript to BioMed Central and take full advantage of:}

- Convenient online submission

- Thorough peer review

- No space constraints or color figure charges

- Immediate publication on acceptance

- Inclusion in PubMed, CAS, Scopus and Google Scholar

- Research which is freely available for redistribution

Submit your manuscript at www.biomedcentral.com/submit
( Biomed Central 